\title{
Chemical profile and antiacetylcholinesterase, antityrosinase, antioxidant and $\alpha$ - glucosidase inhibitory activity of Cynometra cauliflora L. leaves
}

Ado, Muhammad Abubakar and Abas, Faridah and Ismail, Intan Safinar and Mohd Ghazali,

Hasanah and Shaari, Khozirah (2015) Chemical profile and antiacetylcholinesterase, antityrosinase, antioxidant and $\alpha$-glucosidase inhibitory activity of Cynometra cauliflora $L$. leaves. Journal of the Science of Food and Agriculture, 95 (3). pp. 635-642. ISSN 0022-5142; ESSN: 1097-0010

\begin{abstract}
The aim of the current study was (i) to evaluate the bioactive potential of the leaf methanolic extract of Cynometra cauliflora L., along with its respective hexane, dichloromethane, ethyl acetate (EtOAc), n-butanol $(\mathrm{n}-\mathrm{BuOH})$ and aqueous fractions, in inhibiting the enzymes $\alpha$ glucosidase, acetylcholinesterase (AChE) and tyrosinase as well as evaluating their antioxidant activities. (ii) In addition, in view of the limited published information regarding the metabolite profile of $\mathrm{C}$. cauliflora, we further characterized the profiles of the EtOAc and $\mathrm{n}-\mathrm{BuOH}$ fractions using liquid chromatography-diode array detection-electrospray ionization-tandem mass spectrometry. RESULTS: The leaf methanolic extract of $\mathrm{C}$. cauliflora exhibited potent inhibition of all three enzymes and high antioxidant activity. The bioactivity was found to be concentrated in the EtOAc and n-BuOH fractions. A total of 18 compounds were identified in these bioactive fractions, comprising a procyanidin trimer, procyanidin tetramer, procyanidin hexamer, taxifolin pentoside, catechin, vitexin, isovitexin, kaempferol hexoside, quercetin pentoside, quercetin hexoside, apigenin-6-C-glucoside-8-Cglucoside, kaempferol-coumaroyl hexoside and isorhamnetin hexoside. CONCLUSION: The results indicated that $\mathrm{C}$. cauliflora, the leaves in particular, is a rich source of bioactive compounds and could be beneficial for further development of high-value phytomedicinal preparations and functional food products.
\end{abstract}

